\title{
Evidencias de validez del Compendio Internacional de Ítems de Personalidad Abreviado
}

\author{
Evidence of validity of the Mini International Personality Item Pool
}

\author{
Hugo Simkin, a,,*, Lucrecia Borchardt Duter ${ }^{\mathrm{a}}$, Susana Azzollini ${ }^{\mathrm{b}, \mathrm{c}}$ \\ aFacultad de Ciencias Sociales, Universidad de Buenos Aires, Argentina \\ bConsejo Nacional de Investigaciones Científicas y Técnicas, Argentina \\ 'Instituto de Investigaciones, Facultad de Psicología, Universidad de Buenos Aires, Argentina
}

\section{Resumen}

Antecedentes: El modelo de los cinco factores representa uno de los enfoques más empleados para el estudio de la personalidad desde una perspectiva psicológica. Si bien se han relevado numerosos instrumentos para su evaluación, el Compendio Internacional de Ítems de Personalidad Abreviado (Donnellan et al., 2006) constituye una de las principales herramientas destacadas en la literatura. Objetivo: Por este motivo, el propósito del presente estudio consiste en adaptar y validar el instrumento en una muestra no probabilística incidental compuesta por estudiantes de nivel superior en la Ciudad de Buenos Aires $(N=2460)$. Resultado: El análisis factorial confirmatorio indica un buen ajuste a los datos del modelo relevado por los autores, por lo que puede recomendarse su administración en el contexto local. Conclusiones: Se sugiere que futuros trabajos exploren sus propiedades en poblaciones diferentes.

Palabras clave: personalidad; adaptación; validación; análisis factorial confirmatorio; psicometría.

\section{Para citar este artículo:}

Simkin, H., Borchardt, L., \& Azzollini, S. (2020). Evidencias de validez del Compendio Internacional de Ítems de Personalidad Abreviado. Liberabit, 26(1), e320. https://doi.org/10.24265/ liberabit.2020.v26n1.02

\begin{abstract}
Background: the five-factor model represents one of the most widely used approaches to the study of personality from a psychological perspective. Although many instruments have been relied upon for its evaluation, the Mini International Personality Item Pool (Donnellan et al., 2006) is one of the main tools highlighted in the literature. Objective: for this reason, the purpose of the present study is to adapt and validate such instrument in a nonprobability sample composed of university students in Buenos Aires $(N=2460)$. Results: a confirmatory factor analysis indicates a good fit to the data of the model relayed by the authors, so it can be recommended to be administered in the local context. Conclusion: it is suggested that future works explore their properties in different populations.
\end{abstract}

Keywords: personality; adaptation; validation; confirmatory factor analysis, psychometrics.

Este es un artículo Open Access bajo la licencia Creative Commons Atribución-NoComercial-CompartirIgual 4.0

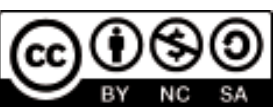

Universidad de San Martín de Porres, Lima - Perú http://ojs3.revistaliberabit.com 


\section{Introducción}

El estudio de la personalidad representa una de las áreas que concentra la mayor cantidad de investigaciones en psicología. Si bien se reconocen diferentes enfoques, el modelo de los cinco factores propuesto por Paul T. Costa y Robert R. McCrae en la década del ochenta se considera uno de los que ha condensado mayor interés en la literatura académica. Para su evaluación, se han construido numerosos instrumentos que varían considerablemente en su longitud oscilando entre los doscientos cuarenta ítems - como en el caso del Inventario de Personalidad NEO Revisado- y los cinco ítems -como en el caso del Inventario de Cinco Ítems de la Personalidad-. Entre las diferentes versiones, uno de los instrumentos que ha despertado mayor interés en la literatura especializada es el Compendio Internacional de Ítems de Personalidad Abreviado (Donnellan et al., 2006), el cual permite relevar las cinco dimensiones a partir de solo veinte ítems. De acuerdo con los autores, la escala resulta ideal para ser administrada cuando no es posible disponer del tiempo necesario para utilizar cuestionarios más extensos. A la vez, resulta uno de los instrumentos empleados con mayor frecuencia para la evaluación del constructo, siendo referenciado en más de mil doscientos artículos académicos según se constata en bases de datos como PsycInfo, ERIC, Pubmed, CAIRN, CLASE, Scielo, Dialnet, Lilacs o Redalyc (Cooper, Smillie, \& Corr, 2010; Martínez-Molina \& Arias, 2018; Oliveira, 2017). Por este motivo, dado que no se han registrado versiones del instrumento en el contexto argentino, el presente estudio se propone su adaptación y validación local.

\section{El modelo de los cinco factores de la personalidad}

El término personalidad deriva etimológicamente de la palabra latina persona y remite a las máscaras utilizadas por los actores en el teatro griego (PérezGarcía \& Bermúdez-Moreno, 2012). En ese contexto, cada máscara correspondía a un tipo de carácter, lo que posibilitaba al público conocer anticipadamente las características o los rasgos principales de la personalidad de cada personaje e inferir el papel que podría desempeñar en la obra (Green, 1994; Wiles, 2007). Desde entonces el término ha sido empleado para referirse a los aspectos más idiosincráticos de una persona que permiten distinguirla de otra (John, Robins, \& Pervin, 2010; McAdams, 1997; McCrae \& Costa, 2012). En la actualidad, la teoría de los rasgos constituye uno de los enfoques más empleados para su estudio (Costa \& McCrae, 2006; Fleeson \& Jayawickreme, 2015; John \& Srivastava, 1999). Desde este enfoque, los rasgos se definen como patrones de pensamientos, emociones y comportamientos que se mantienen relativamente estables a lo largo del ciclo vital (Costa \& McCrae, 1980b; Kassin, 2003). Aunque diferentes modelos han intentado clasificar la multiplicidad de rasgos de la personalidad en diferentes categorías (Ashton \& Lee, 2001; Cattell, 1950; Eysenck \& Eysenck, 1976; Piedmont, 1999), el modelo de los cinco factores (Five Factor Model, en adelante FFM, Costa \& McCrae, 1980b) se destaca entre los que han cobrado mayor aceptación (McCrae \& Costa, 2013; McCrae \& Sutin, 2018).

Cabe señalar que la estructura pentafactorial fue el resultado de investigaciones empíricas realizadas por diversos autores a partir de las cuales se arribó a un consenso acerca de una taxonomía general de los rasgos de la personalidad (Bond, 1994; John, Naumann, \& Soto, 2008). Esa taxonomía se organiza en cinco factores llamados (1) apertura a la experiencia, (2) responsabilidad, (3) extraversión, (4) amabilidad y (5) neuroticismo.

\section{Apertura a la experiencia}

Históricamente la apertura a la experiencia o apertura mental ha sido uno de los factores que ha generado mayor controversia, debido a cierto desacuerdo en relación a las facetas que lo componen (Connelly, Ones, \& Chernyshenko, 2014; Digman, 1990; Fiske, 1949). En la literatura, usualmente se emplea el término para distinguir a las personas creativas (Li et al., 2015; McCrae, 1987), con variados intereses artísticos (Feist \& Brady, 2004) e intelectuales (George \& Zhou, 2001; LePine, Colquitt, 
\& Erez, 2000; Woo et al., 2014), y una marcada tendencia a la búsqueda de estimulación (Aluja, García, \& García, 2003) y a la flexibilidad cognitiva (Silvia, Nusbaum, Berg, Martin, \& O’Connor, 2009). Además, el factor supone un fuerte vínculo con la percepción de emociones y, si bien suele asociarse a la experiencia de sentimientos positivos, algunas de sus facetas pueden relacionarse con emociones negativas, como la tristeza o la desesperanza (Carrillo, Rojo, SánchezBernardos, \& Avia, 2001; Wolfestein \& Trull, 1997). De acuerdo con Khoo y Simms (2018), las personas que obtienen un puntaje alto en la faceta 'sentimientos' son más propensas a experimentar una gama más amplia de emociones, tanto positivas como negativas, lo que puede incluir emociones relacionadas con la depresión. Asimismo, aquellos individuos con puntajes altos en la faceta 'fantasía' podrían experimentar una mayor discrepancia entre el estado idealizado de sí mismos y del mundo y el estado real (Khoo \& Simms, 2018). Por otra parte, se ha observado que la apertura se encuentra vinculada a la ideología política (Lee, Ashton, Ogunfowora, Bourdage, \& Shin, 2010; Hiel, Kossowska, \& Mervielde, 2000; Hiel \& Mervielde, 2004). En particular, una alta apertura a la experiencia se asocia a un menor autoritarismo del ala de derechas y a la inclinación por los partidos que promueven valores de izquierda (Barbaranelli, Caprara, Vecchione, \& Fraley, 2007; Duckitt \& Sibley, 2010; Lee et al., 2010). Por este motivo, los individuos con mayor apertura se muestran más receptivos frente a la diversidad sexual (Hong \& Rust, 1989; Zoeterman \& Wright, 2014) y presentan menores prejuicios raciales, étnicos y religiosos (Cullen, Wright, \& Alessandri, 2002; Flynn, 2005; Silvia \& Sanders, 2010). Además, la apertura se relaciona con la capacidad para desarrollar un pensamiento propio, diferente del de el grupo de referencia, y a la habilidad para expresar abiertamente esta disidencia (Packer, 2010). Estas características de la apertura responden, en parte, a la facilidad para el pensamiento simbólico y la consecuente posibilidad de abstracción, alejada de la experiencia concreta (McCrae \& Sutin, 2009). Dependiendo de las habilidades intelectuales específicas del individuo, el pensamiento simbólico puede facilitar el uso de la matemática, el lenguaje, el dominio de la música o de las artes visuales (McCrae \& Costa, 1997, 2012).

Por el contrario, las personas con bajos niveles de apertura a la experiencia generalmente poseen poca curiosidad intelectual, prefiriendo lo claro y concreto sobre lo abstracto, ambiguo y complejo (McCrae \& Sutin, 2009). Este aspecto conlleva por lo general a evidenciar desinterés por las artes y las ciencias, argumentando que tales actividades carecen de uso práctico (Costa, McCrae, \& Holland, 1984; Feist, 1998), y a preferir lo conocido a la novedad debido a su resistencia al cambio (Anderson, John, \& Keltner, 2012; Dollinger, 1993). Tal apego a lo conocido contribuye a explicar las asociaciones entre una baja apertura y cierto rechazo por aquellos individuos que se perciben como diferentes por su etnia, religión u orientación sexual (Ekehammar, Akrami, Gylje, \& Zakrisson, 2004; Hodson, Hogg, \& MacInnis, 2009; Perry \& Sibley, 2013) y a la inclinación por los valores conservadores que promueven los partidos del ala de derecha (Butler, 2000; Heaven \& Bucci, 2001; Jost, Nosek, \& Gosling, 2008).

$\mathrm{Al}$ igual que Connelly et al. (2014), McCrae y Sutin (2009) señalan que el constructo resulta difícil de comprender debido a que las diferentes facetas de la apertura no guardan una relación estricta, motivo por el cual ciertas personas pueden presentar algunas características del factor y no otras. Por ejemplo, tal como sugieren los autores, una persona puede experimentar una amplia sensibilidad estética pero mostrarse autoritario, dogmático, cerrado e intolerante en cuanto a valores políticos distintos de los suyos. Además, diferentes estudios observan que resulta difícil distinguir la apertura de otros constructos psicológicos, como en el caso de la inteligencia (Harris, 2004; McCrae et al., 2008). En este sentido se ha sugerido, sin embargo, que ambos reflejan constructos diferentes, por lo que bajos niveles de apertura no necesariamente suponen bajos niveles de inteligencia (Borders, 2012; McCrae, 1993, 1994). 
Finalmente, si bien la elevada apertura ha sido valorada positivamente en la literatura, altas puntuaciones en este factor también fueron evaluadas de manera negativa. Por ejemplo, personas con mayor apertura encuentran dificultades para cumplir objetivos y metas, debido a que suelen dispersarse generando constantemente nuevas ideas (Piedmont, Sherman, \& Sherman, 2012). Por el contrario, el pragmatismo característico de una baja apertura puede promover el desarrollo de la responsabilidad, un mayor autocontrol y eficacia en la resolución de problemas (Dollinger, Leong, \& Ulicni, 1996). Además, dado que las personas con baja apertura tienden a prestar poca atención a sus emociones, perciben en menor medida la ansiedad que suele generar la presión en el cumplimiento de objetivos (Griffin \& Hesketh, 2004).

\section{Responsabilidad}

De acuerdo con Roberts, Jackson, Fayard, Edmonds y Meints (2009), el factor responsabilidad presenta una larga tradición en la literatura académica psicológica, como es el caso de la noción de superyó en Freud, los conceptos subsidiarios del yo ideal y de la consciencia, y disposiciones asociadas a la necesidad de realización y el control. Diferentes autores sugieren que el aspecto central de la responsabilidad consiste en la tendencia a adecuarse a las normas sociales prescriptas, controlar los impulsos, dirigirlos a una meta, planificar y a la capacidad de postergar la gratificación (Costa \& McCrae, 1996; John \& Srivastava, 1999; Roberts et al., 2009).

La literatura especializada suele valorar de manera positiva una elevada responsabilidad y de manera negativa una responsabilidad baja. Por ejemplo, se ha sugerido que las personas con mayor responsabilidad tienden a resultar exitosas a través de la planificación deliberada y de la persistencia en el logro de sus objetivos (Anderson et al., 2012; McCrae \& Costa, 2012; Roberts et al., 2009). Por ello, suelen ser consideradas por los demás como inteligentes y confiables (Huang et al., 2017; McCrae \& Costa, 2012; Steptoe, Easterlin, \& Kirschbaum,
2017). Por el contrario, los individuos con baja responsabilidad suelen ser evaluados de manera negativa por sus pares (Smith, Barstead, \& Rubin, 2017) y evidenciarían un comportamiento impulsivo que podría dañar a otros individuos tanto como a la propia persona (Arthur \& Graziano, 1996; John, Robins et al., 2010; Steptoe et al., 2017). Por este motivo, algunos autores señalan que si bien a menudo la impulsividad produce recompensas inmediatas, puede traer consecuencias no deseadas a largo plazo (Cao, Su, Liu, \& Gao, 2007; Whiteside \& Lynam, 2001). Asimismo, actuar de forma impulsiva no permitiría contemplar estrategias alternativas frente a un mismo problema que pudieran resultar más eficaces (Costa \& McCrae, 1996).

Sin embargo, si bien la responsabilidad suele tener una valoración positiva en la literatura psicológica, se han identificado tanto aspectos considerados negativos de una alta responsabilidad, como aspectos positivos ligados a las puntuaciones bajas. Por ejemplo, los individuos con elevada responsabilidad podrían resultar perfeccionistas, compulsivos o adictos al trabajo (McCrae \& Costa, 2012). Por su parte, la impulsividad propia de la baja responsabilidad no resultaría intrínsecamente negativa, ya que en ciertas situaciones donde se requiere tomar decisiones de manera rápida, actuar de manera impulsiva podría ser una respuesta eficaz (Anderson et al., 2012).

\section{Extraversión}

El término extraversión fue difundido en el campo psicológico a principios del siglo XX por Jung (1923, 1939) para distinguir a las personas focalizadas en el mundo exterior o extravertidas de las personas focalizadas en su propio mundo interno o introvertidas. Posteriormente, Eysenck (1952) adoptó el término para referirse a uno de los factores del modelo de la personalidad. Si bien inicialmente surgieron ciertos debates respecto de su dimensionalidad (Carrigan, 1960), actualmente se considera uno de los factores que ha demostrado mayor consistencia (McCrae \& Costa, 2008, 2012). 
Las personas que poseen altos niveles de extraversión tienden a buscar la estimulación social y la interacción interpersonal (Costa \& McCrae, 1996). Estos individuos a menudo se describen como alegres, llenos de energía y proclives a la conformación de grupos (Anderson et al., 2012; Costa \& McCrae, 1980a; Williams, 1992). Asimismo, la extraversión se relaciona con una visión relativamente positiva del mundo, dado que las personas extravertidas juzgan eventos neutrales de manera más positiva que la mayoría de los individuos (Uziel, 2006; Wilt \& Revelle, 2009). Por este motivo, diferentes autores observan que suelen experimentar emociones positivas con más frecuencia (Costa \& McCrae, 1980b; Lucas \& Baird, 2004; Watson \& Clark, 1992, 1997). Dado su carácter gregario, las personas extravertidas se destacan en actividades en donde se requiere interacción con otros, siendo menos propensas a experimentar ansiedad o miedo a ser evaluadas negativamente por el entorno (McCrae \& Costa, 2012).

Por su parte, las personas introvertidas tienden a ser tranquilas, reservadas y suelen involucrarse relativamente poco en situaciones sociales (Costa \& McCrae, 1996), prefiriendo dedicarse a tareas más solitarias (Costa \& McCrae, 1996; McCrae \& Costa, 2012). A pesar de que se han relevado antecedentes que asocian la introversión con síntomas patológicos, un aspecto a destacar es que las personas introvertidas no necesariamente tienen temor a las situaciones sociales, como es el caso de la timidez, sino que prefieren pasar más tiempo en soledad, prescindiendo de estimulación social (Anderson et al., 2012). De esta manera, si bien la extraversión se relaciona con el afecto positivo, no es la introversión sino el neuroticismo el factor predictor del afecto negativo (Magnus, Diener, Fujita, \& Pavot, 1993; Rusting \& Larsen, 1997).

De acuerdo con Borders (2012), la extraversión suele, con frecuencia, confundirse con sociabilidad. Sin embargo, el autor observa que los individuos con altos niveles de extraversión presentan tanto aspectos asociados a la calidez como a la dominación. Por este motivo, diferentes estudios han sugerido que la característica básica de esta dimensión no sería meramente la sociabilidad sino la disposición a presentar un comportamiento social activo o dominante (Ashton, Lee, \& Paunonen, 2002; McCrae \& John, 1992).

Finalmente, aunque la introversión ha presentado una valoración negativa en la literatura, recientemente se ha observado que puede relacionarse con la percepción de experiencias emocionales positivas (Hills \& Argyle, 2001b). Por el contrario, se han señalado elementos comunes entre la extraversión y el comportamiento antisocial (Allsopp \& Feldman, 1974), el narcisismo (Ong et al., 2011), el juego patológico (Roy, Jong, \& Linnoila, 1989), el consumo excesivo de alcohol (Martsh \& Miller, 1997), comportamientos riesgosos (Miller et al., 2004), entre otras características valoradas negativamente por la literatura especializada.

\section{Amabilidad}

El concepto de amabilidad se remonta a la antigüedad, encontrándose en la literatura innumerables referencias al valor de la cooperación con otros individuos en las relaciones sociales (e.g., Akrasia de Aristóteles) (Graziano \& Tobin, 2009). Sin embargo, el modelo de los cinco factores es uno de los primeros en presentarla como un factor de la personalidad (Digman \& Takemoto-Chock, 1981). De acuerdo con la literatura, tal altruismo representa una de las características principales de esta dimensión (Carlo, Okun, Knight, \& Guzman, 2005; Graziano, Habashi, Sheese, \& Tobin, 2007).

Las personas con altos niveles de amabilidad suelen ser apreciadas por sus pares y superiores, entre otras razones, porque tienden a cooperar con otros y a excusarse por las fallas de los demás (Graziano \& Tobin, 2009; Huang et al., 2017). En este sentido, se ha observado que la amabilidad puede modular la relación entre la responsabilidad y la efectividad en el cumplimiento de objetivos en el trabajo grupal (Bradley, Baur, Banford, \& Postlethwaite, 2013; Witt, Burke, Barrick, \& Mount, 2002). Personas agradables poseen 
una visión optimista de la naturaleza humana, considerando que la gente es esencialmente honesta, decente y digna de confianza (Graziano, 1994; McCrae \& Costa, 2012). Al igual que ocurre con la apertura, diferentes autores han observado que las personas amables suelen inclinarse por aquellos partidos que reflejen valores más cercanos a las ideologías de izquierda (Barbaranelli et al., 2007; Caprara, Vecchione, \& Schwartz, 2009; Hirsh, DeYoung, Xu, \& Peterson, 2010) y presentan actitudes positivas hacia grupos socialmente estigmatizados (e.g., homosexuales, inmigrantes) (Butrus \& Witenberg, 2013; Duckitt \& Sibley, 2010; Sibley \& Duckitt, 2009). Consecuentemente, se ha señalado que la amabilidad está relacionada negativamente a la competitividad y al conflicto intergrupal (Suls, Martin, \& David, 1998), en tanto estos individuos suelen transformar las situaciones competitivas en cooperativas (Graziano, Jensen-Campbell, \& Hair, 1996). Diferentes estudios señalan que esta eficacia en la resolución de los conflictos interpersonales puede deberse a su habilidad para tolerar emociones negativas, como la frustración o la ira (Bresin, Hilmert, Wilkowski, \& Robinson, 2012; Haas, Omura, Constable, \& Canli, 2007; Meier \& Robinson, 2004), lo que presupone además una marcada capacidad para perdonar (Lee \& Ashton, 2012; Steiner, Allemand, \& McCullough, 2012; Strelan, 2007).

Por su parte, los individuos con bajos niveles de amabilidad suelen anteponer su propio interés por encima del de otros, mostrándose indiferentes hacia el bienestar de los demás (Costa \& McCrae, 1996). Muchas veces, su escepticismo acerca de la benevolencia de las personas los conduce a ser desconfiados, pudiendo parecer antipáticos o poco cooperativos (Anderson et al., 2012).

Cabe destacar que, de acuerdo con Borders (2012), existe en la literatura un enfoque reduccionista del factor amabilidad que lo resume en buenos vs. malos. Por este motivo se ha señalado que resulta más apropiado referirse a la polaridad de la amabilidad en calidad de prosocial vs. antisocial (Borders, 2012;
McCrae \& John, 1992). A la vez, a pesar de que la elevada amabilidad suele tener una connotación positiva en la literatura, se ha sugerido que también puede presentar una forma patológica, caracterizada por una marcada dependencia de los demás (Costa \& McCrae, 1985; Samuel \& Gore, 2012; Widiger, 2009). Asimismo, la tendencia al acuerdo puede dificultar el proceso de toma de decisiones cuando estas requieren actuar con celeridad (McCrae \& Costa, 2012). Finalmente, debido a los estereotipos de género, en ciertos contextos la elevada amabilidad puede ser valorada socialmente de manera negativa en los varones (Judge, Livingston, \& Hurst, 2012).

\section{Neuroticismo}

Históricamente el neuroticismo representa uno de los factores más relevantes en el estudio de la personalidad (McCrae \& Costa, 2012) y existen pocos modelos que no lo incluyen (Digman, 1990). Widiger (2009) señala que el término neurosis fue introducido por William Cullen en 1769 para referirse a desórdenes resultantes de una «afección general» del sistema nervioso. Posteriormente, Freud (1933) lo emplea para describir una afección caracterizada por trastornos mentales, sufrimiento emocional e incapacidad de hacer frente eficazmente a las exigencias «normales» de la vida. Asimismo, indica que la mayoría de los individuos poseen signos de neurosis, los cuales difieren en cuanto al grado de sufrimiento y a los síntomas específicos de la angustia.

En la actualidad, el término se emplea dentro del modelo de los cinco factores para referirse a la tendencia a experimentar emociones negativas como miedos, sentimientos de culpa, tristeza o enojo (Costa, McCrae, \& Arenberg, 1980; McCrae \& Costa, 2008). Diferentes estudios sugieren que las personas con altos niveles de neuroticismo poseen menores recursos de afrontamiento para sobreponerse al estrés ambiental (Gunthert, Cohen, \& Armeli, 1999; Smith et al., 2017; Suls et al., 1998), son más propensas a interpretar las situaciones ordinarias como una amenaza y consideran tareas que para otros generalmente suelen ser sencillas 
como particularmente difíciles (Anderson et al., 2012; Goldenberg, Pyszczynski, McCoy, Greenberg, \& Solomon, 1999; Magnus et al., 1993). Por este motivo, las personas con un alto nivel de neuroticismo son emocionalmente reactivas y responden afectivamente a eventos que no afectan a la mayoría de los individuos, reaccionando de manera más intensa de lo normal (Anderson et al., 2012; Costa \& McCrae, 1987; Jeronimus, Riese, Sanderman, \& Ormel, 2014). Estos problemas en la regulación emocional pueden afectar la capacidad de pensar con claridad y de tomar decisiones (McCrae \& Costa, 2012; Perkins, Arnone, Smallwood, \& Mobbs, 2015). De acuerdo con Rammstedt (2007), una característica distintiva del neuroticismo radica en que las rumiaciones y las preocupaciones se encuentran particularmente centradas en el sí mismo. Para el autor, no es solo que las personas con mayor neuroticismo se preocupen sustancialmente más que otros, sino que además tienden a preocuparse exclusivamente por ellas mismas antes que por problemáticas sociales o comunitarias.

Si bien el FFM no evalúa patologías mentales, altos niveles en el factor neuroticismo constituyen un riesgo en el desarrollo de ciertos trastornos psicológicos (Khan, Jacobson, Gardner, Prescott, \& Kendler, 2005; Ormel, Rosmalen, \& Farmer, 2004; Trull \& Durrett, 2005). Diferentes trabajos han reportado asociaciones entre el neuroticismo y la depresión (Jylhä \& Isometsä, 2006; Yoon, Maltby, \& Joormann, 2013), la ansiedad (Paulus, Vanwoerden, Norton, \& Sharp, 2016), el trastorno de estrés postraumático (Breslau \& Schultz, 2013), los trastornos alimentarios (Heaven, Mulligan, Merrilees, Woods, \& Fairooz, 2001), entre otros trastornos mentales (Hettema, Neale, Myers, Prescott, \& Kendler, 2006; Krueger, Caspi, Moffitt, Silva, \& McGee, 1996; Ormel et al., 2013).

Por su parte, los individuos con un bajo neuroticismo o con una alta estabilidad emocional suelen experimentar una baja frecuencia de emociones negativas (Costa, McCrae, \& Norris,
1981; Hills \& Argyle, 2001a). De acuerdo con Walker y Gorsuch (2002), esta característica puede explicarse en parte porque aquellas personas con más estabilidad tienen una mayor capacidad de perdonar a otras y de perdonarse a ellas mismas. Así, aquellas personas con altos puntajes en estabilidad emocional y en responsabilidad suelen correr un menor riesgo de padecer diversos problemas relacionados con la ansiedad, como el bruxismo, el tabaquismo o la obesidad, lo que promueve una mayor longevidad (Judge, Vianen, \& Pater, 2004; Sutin, Terracciano, Ferrucci, \& Costa, 2010). Además, al igual que les sucede a los individuos con baja apertura, la ausencia de emociones negativas contribuye a incrementar la eficacia en alcanzar sus metas y objetivos (Judge \& Erez, 2007; Kaiser \& Ozer, 1997; Rothmann \& Coetzer, 2003).

Sin embargo, aunque el factor ha presentado una valoración negativa en la literatura, también se han identificado relaciones entre el neuroticismo y características valoradas de manera más positiva, como la creatividad (Clark \& DeYoung, 2014; Nowakowska, Strong, Santosa, Wang, \& Ketter, 2005; Srivastava \& Ketter, 2010).

\section{Criticas al modelo de los cinco factores de la personalidad}

Una de las críticas que ha recibido el modelo de los cinco factores de la personalidad remite a su estructura factorial. Por un lado, distintos estudios han reportado factores de segundo orden que agrupan en un primer factor a la amabilidad, la responsabilidad $\mathrm{y}$ al neuroticismo; y en un segundo factor, a la extroversión y a la apertura (Dermody et al., 2016; DeYoung, 2006; Digman, 1997; Simkin \& PérezMarín, 2018). Por otra parte, se ha sugerido la necesidad de incorporar nuevos factores al modelo, tales como la espiritualidad o la honestidad (Ashton \& Lee, 2001; Cheung, Cheung, Leung, Ward, \& Leong, 2003; Piedmont, 1999).

Una segunda crítica remite a sus limitaciones explicativas. Al tratarse de un enfoque de carácter 
descriptivo, el modelo no permite comprender el modo en que se construyen y desarrollan los rasgos ni la forma en la que estos podrían afectar a otros constructos psicológicos (McCrae \& Costa, 1996, 2010). La teoría de los cinco factores, que mediante una serie de postulados describe lo que los autores denominan sistema de la personalidad, fue propuesta precisamente por McCrae y Costa (1996) buscando superar algunas de estas dificultades. En ella, los autores plantean el modo en que los cinco factores interactúan con el ambiente y con la cultura dando lugar a características adaptativas (McCrae \& Costa, 2012). Sin embargo, se ha observado que la propuesta aún requiere de mayor apoyo tanto teórico como empírico para sortear las limitaciones señaladas (Laher, 2013).

Por último, una de las principales críticas que recibe este tipo de modelos de la personalidad radica en que tiende a adoptar un enfoque ahistórico e individualista que favorece el mantenimiento del status quo (Prilleltensky, 2003; Sloan, 1997, 2009; Venn, 1984).

\section{Método}

\section{Diseño}

Para el presente estudio se ha optado por un diseño de tipo instrumental (Ato, López, \& Benavente, 2013; Montero \& León, 2007).

\section{Participantes}

La muestra, de tipo intencional, incluye a 2460 estudiantes de nivel superior de la ciudad de Buenos Aires con edades que oscilan entre los 18 y 35 años $(M=23.58 ; D E=4.05)$ y de ambos sexos (hombres $=42.4 \%$; mujeres $=57.6 \%$ ). Se consideraron como criterios de exclusión contar con diagnóstico psiquiátrico y con discapacidad sensorial, motriz o intelectual debido a la imposibilidad de brindar los apoyos necesarios para la plena participación de quienes cumplen con tales criterios.

\section{Instrumentos}

Compendio Internacional de Ítems de Personalidad Abreviado (Mini International Personality Ítem Pool, Mini-IPIP). El Mini-IPIP (Donnellan et al., 2006) es un cuestionario autoadministrable de 20 ítems con un formato tipo Likert con cinco anclajes de respuesta en función del grado de acuerdo de los participantes, siendo 1 "Completamente en desacuerdo" y 5 «Completamente de acuerdo». La escala evalúa cinco dimensiones de la personalidad en población adolescente y adulta: (1) apertura a la experiencia o apertura mental (Openness to experience; items 5, 10, 15, 20) (e.g., «No me interesan las ideas abstractas»); (2) responsabilidad, escrupulosidad o tesón (Conscientiousness; items 3, 8, 13, 18) (e.g., «Soy algo desordenado/a»); (3) extraversión (Extraversion; items 1, 6, 11, 16) (e.g., «No me gusta llamar la atención»); (4) amabilidad, afabilidad o tendencia al acuerdo (Agreeableness; items 2, 7, 12, 17) (e.g., «No me interesan mucho los problemas de los demás»); y (5) neuroticismo o estabilidad emocional (Neuroticism, Emotional Stability; items 4, 9, 14, 19) (e.g., «Raras veces me siento triste»). Los cinco factores han presentado propiedades psicométricas aceptables de acuerdo a lo informado por los autores de la prueba original y por estudios posteriores (Donnellan et al., 2006; Cooper, Smillie, \& Corr, 2010). La adaptación al español del MiniIPIP (ver anexo) fue realizada de acuerdo con los estándares metodológicos internacionales sugeridos por la International Test Commission (ITC) para la adaptación de un instrumento a distintos contextos idiomáticos (Muñiz, Elosua, \& Hambleton, 2013; Muñiz \& Hambleton, 2000).

Cuestionario de datos sociodemográficos. Se elaboró un cuestionario ad hoc que solicitaba a los participantes consignar la edad, el género y el nivel de estudios. 


\section{Procedimiento}

El estudio se realizó en una universidad pública de la Ciudad Autónoma de Buenos Aires. Los estudiantes fueron invitados a colaborar voluntariamente en el proyecto de investigación en el que se enmarca el presente estudio, solicitándoles su consentimiento. Aquellos que participaron respondieron individualmente en la universidad y en aquellos horarios de clases cedidas por las Cátedras que colaboraron con el presente estudio. A lo largo de la administración de los cuestionarios, los investigadores permanecieron presentes. Antes de responder los cuestionarios, se les hizo constar que los datos derivados de la investigación se utilizarían con fines exclusivamente académico-científicos bajo la Ley Nacional 25.326 de protección de los datos personales. Una vez completados los instrumentos de evaluación, estos fueron entregados a los investigadores en un sobre cerrado con el propósito de respetar el anonimato de los participantes.

\section{Análisis de datos}

Los análisis estadísticos fueron realizados empleando los programas SPSS 22 y EQS 6.1. Dada la naturaleza ordinal de los ítems, se optó por realizar el análisis de los datos empleando una matriz de correlaciones policóricas. En primer lugar, se analizó la confiabilidad del Mini-IPIP a partir del análisis de consistencia interna, empleando el coeficiente omega de McDonald siguiendo recomendaciones de la literatura (Dunn, Baguley, \& Brunsden, 2014). En segundo lugar, la validez de constructo fue evaluada a partir de realizar un análisis factorial confirmatorio (AFC), teniendo en cuenta el método GLS robusto, tal como ha sido sugerido en los antecedentes (Schermelleh-Engel, Moosbrugger, \& Müller, 2003). El modelo fue evaluado posteriormente mediante los índices de bondad de ajuste $\chi^{2}$, NFI (Normed Fit Index), NNFI (Non-Normed Fit Index), CFI (Comparative Fit Index), IFI (Incremental Fit Index) y el índice RMSEA (Root Mean Square Error of Approximation), siguiendo lo sugerido en los antecedentes (Holgado-Tello, Chacón-Moscoso, Barbero-García, \& Vila-Abad, 2009; Schumacker \& Lomax, 2004). Finalmente, se procedió a realizar un estudio de invarianza factorial con el propósito de verificar que el modelo no presente un funcionamiento diferencial de acuerdo al género de los participantes (Davidov, Schmidt, Billiet, \& Meuleman, 2018).

\section{Resultados}

En primer lugar, se evaluó la consistencia interna de los diferentes dominios a partir del coeficiente omega, arrojando resultados aceptables de acuerdo a la literatura, a partir de la cual se consideran adecuados aquellos valores superiores a .70 (CampoArias \& Oviedo, 2008; Dunn et al., 2014; Green \& Yang, 2015).

Tabla 1

Coeficiente omega de los diferentes dominios del Mini-IPIP

\begin{tabular}{cccccc}
\hline & $\mathrm{O}$ & $\mathrm{C}$ & $\mathrm{E}$ & $\mathrm{A}$ & $\mathrm{N}$ \\
\hline$\omega$ & .88 & .80 & .82 & .88 & .77 \\
\hline
\end{tabular}

Nota: $* \mathrm{O}=$ Apertura; $\mathrm{C}=$ Responsabilidad; $\mathrm{E}=$ Extraversión; $\mathrm{A}=$ Amabilidad; $\mathrm{N}=$ Neuroticismo. 
En segundo lugar, luego de analizar la consistencia interna, se procedió a evaluar la validez de constructo a partir de la realización del AFC. Tal como se observa en la Tabla 2, el ajuste del modelo fue valorado mediante los índices de bondad de ajuste $\chi^{2}$, NFI, NNFI, CFI, IFI y RMSEA, siguiendo recomendaciones previas (Holgado-Tello et al., 2009; Kline, 2005, 2010; Schumacker \& Lomax, 2004). Los resultados dan cuenta de un buen ajuste de los datos empíricos en relación al modelo teórico de los cinco factores de la personalidad (Bentler, 1990; Hu \& Bentler, 1999), arrojando valores superiores a .90, valor considerado en la literatura como punto de corte para distinguir un ajuste adecuado (Brown, 2015). Por su parte, el índice RMSEA alcanzó valores entre 0 y .08 , considerados adecuados en la literatura especializada (Lévy \& González, 2006).

Tabla 2

Análisis Factorial Confirmatorio del Mini-IPIP. Índices de ajuste

\begin{tabular}{ccccccc}
\hline & $\chi_{(\mathrm{gl})}^{2}$ & NFI & NNFI & CFI & IFI & RMSEA (IC) \\
\hline Ipip* & $1017.6242_{(160)}$ & .914 & .913 & .927 & .927 & $.061(.058 ; .065)$ \\
\hline
\end{tabular}

Nota: * Modelo pentafactorial.

En la Figura 1 puede observarse el modelo de cinco factores del Compendio Internacional de Ítems de Personalidad Abreviado Mini-IPIP propuesto por Donnellan et al. (2006).

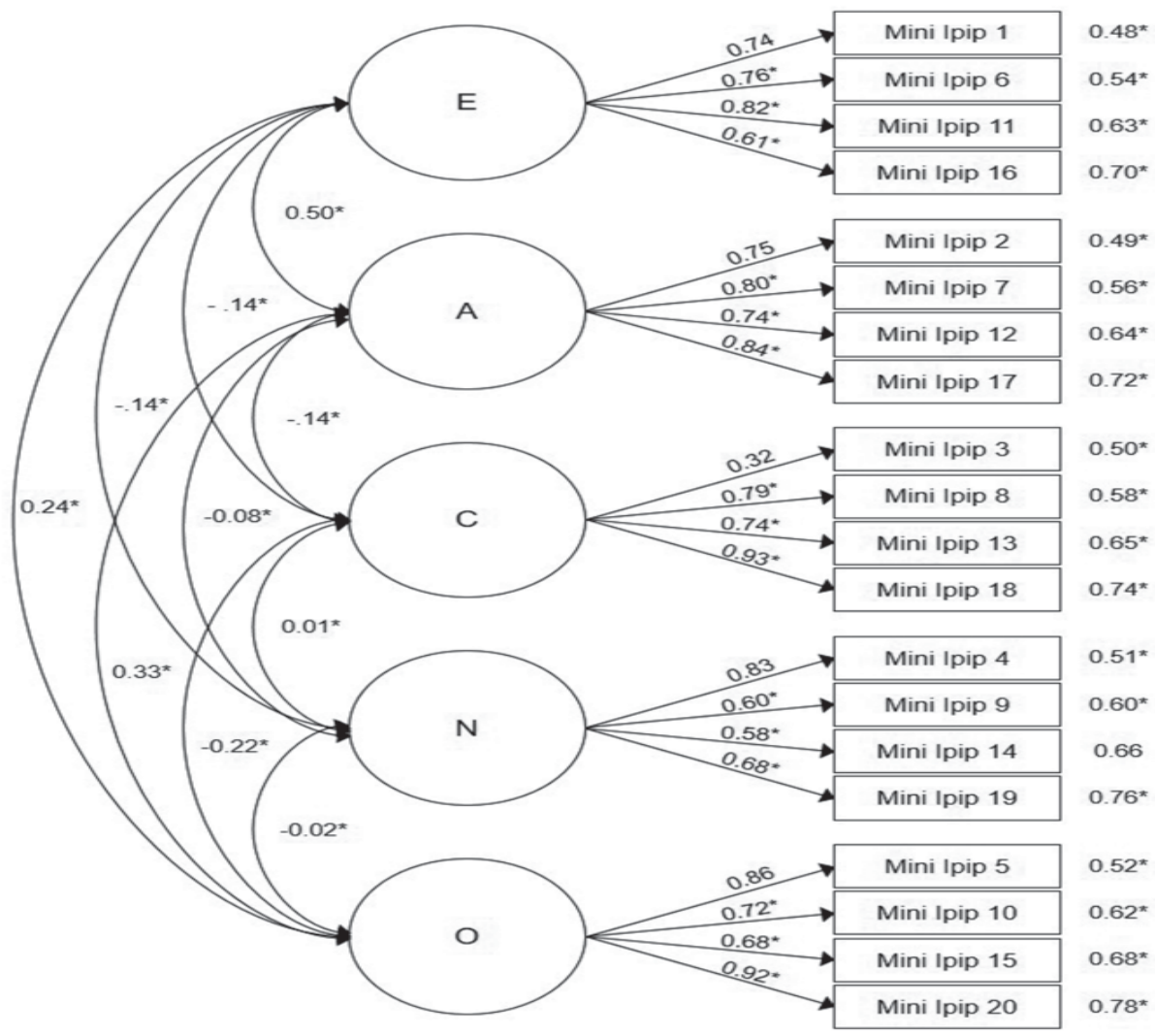

Figura 1. Modelo estructural del Mini-IPIP y betas estandarizados del AFC. Fuente: elaboración propia 
Finalmente, se realizó un estudio de invarianza factorial para verificar que el modelo no presente un funcionamiento diferencial de acuerdo con la variable género. El análisis de la invarianza factorial se efectúa a partir de la comparación de los índices CFI y RMSEA. Como puede observarse en la Tabla 3, las diferencias halladas entre los CFI fueron iguales o inferiores a .01, mientras que las diferencias registradas para RMSEA fueron menores a .015, dando cuenta de que no existen diferencias significativas entre los modelos testados con distintos niveles de restricción al segmentar la muestra según la variable género (Barrera-Barrera, Navarro-García \& Peris-Ortiz, 2015; Davidov et al., 2018).

Tabla 3

Análisis de Invarianza Factorial del Mini-IPIP

\begin{tabular}{lcccccc}
\hline & S-B & gl & RMSEA [IC 90\%] & $\Delta$ RMSEA & CFI & $\Delta$ CFI \\
\hline Modelo configural & 1524.54 & 310 & $.057[.054-.059]$ & - & .940 & - \\
Modelo métrico & 1732.41 & 330 & $.059[.056-.062]$ & -.002 & .930 & .01 \\
Modelo estructural & 1727.47 & 340 & $.058[.055-.060]$ & -.001 & .931 & .009 \\
\hline
\end{tabular}

Nota: $\triangle$ RMSEA = diferencia de RMSEA; $\Delta$ CFI = diferencia de CFI.

\section{Discusión}

A partir de los resultados del presente estudio puede observarse que el Compendio de Ítems Internacional de Personalidad Abreviado o Mini International Personality Ítem Pool (Mini-IPIP) presenta propiedades psicométricas aceptables en el contexto local. El instrumento incluye veinte ítems similares a los propuestos por los autores originales (Donnellan et al., 2006). Si bien este tipo de instrumentos breves no brindan una evaluación concluyente de la personalidad de un individuo en particular, pueden ofrecer una aproximación general al constructo, especialmente en cuanto a su relación con otras variables de interés (Donnellan et al., 2006; McAdams, Shiner, \& Tackett, 2019). Los índices de fiabilidad alcanzan valores equivalentes a los reportados por otros trabajos en el contexto internacional en poblaciones similares (Baldasaro, Shanahan, \& Bauer, 2013; Cooper et al., 2010; Laverdière, Morin, \& St-Hilaire, 2013; Li, Sang, Wang, \& Shi, 2012; Oliveira, 2017). Del mismo modo, de acuerdo con el AFC, el modelo pentafactorial se ajusta a los datos recabados en campo, tal como se ha señalado en la literatura (Costa \& McCrae, 1980b; Digman \& Takemoto-Chock, 1981; Norman, 1963; Tupes \& Christal, 1961).
Cabe señalar que los valores inferiores a .50 de las covarianzas entre las variables latentes podrían ser considerados como evidencia de validez discriminante, conduciendo a evaluar la posibilidad de incluir nuevas dimensiones al modelo (Lévy \& Varela, 2006). Si bien se ha observado que los rasgos se encuentran organizados jerárquicamente en solo cinco niveles de organización superior (Heuchert, Parker, Stumpf, \& Myburgh, 2000; McCrae \& John, 1992; Piedmont \& Chae, 1997), McCrae y Costa (2012) han señalado que estos representan la evidencia disponible al momento, lo que posibilitaría expandir el modelo incluyendo nuevos factores. En este sentido, los resultados del presente estudio se encuentran en línea con diferentes autores que han propuesto alternativas al FFM, contemplando la incorporación de nuevas dimensiones (Ashton \& Lee, 2001; Cheung et al., 2003; Piedmont, 1999). Por este motivo, tal como ha sido sugerido en nuestro contexto (Simkin, 2017; Simkin \& Pérez-Marín, 2018), se recomienda que futuras investigaciones continúen explorando la estructura factorial del modelo de los cinco factores de la personalidad.

Por su parte, con excepción de los ítems 3, 14, 15 y 16, los parámetros estimados alcanzaron los 
valores aceptables, de acuerdo con la literatura especializada ( $\geq .70$ ), considerando que las cargas factoriales en los estudios psicológicos tienden oscilar en torno a .50 (Beauducel \& Herzberg, 2006).

Por último, se destaca que el análisis de invarianza factorial permitió verificar que no existen diferencias significativas entre los modelos testados con distintos niveles de restricción al segmentar la muestra según el género de los participantes. Este tipo de análisis aporta mayor robustez al instrumento, contribuyendo a sostener la replicabilidad de los datos en muestras diferentes, detectando un funcionamiento independiente del tipo de muestra (Meredith, 1993). Si bien para la evaluación del modelo de los cinco factores la mayoría de los estudios analizan la invarianza factorial empleando el género como variable (Chiorri, Marsh, Ubbiali, \& Donati, 2016; Samuel, South, \& Griffin, 2015), futuros trabajos deberían aportar mayor evidencia de la invarianza factorial del modelo considerando variables alternativas de segmentación, como el nivel educativo o la adscripción religiosa.

El presente estudio aporta técnicas válidas y confiables para la evaluación de la personalidad en el contexto argentino desde el modelo de los cinco factores, aunque se destaca la necesidad de que futuros trabajos puedan contar con muestras representativas y explorar las propiedades psicométricas de la escala en población general. Cabe señalar que emplear el modelo de los cinco factores no implica desconocer las críticas que este ha recibido ni sus implicancias. En efecto, sostenemos que el análisis de la personalidad basado en la descripción de los rasgos que realiza dicho modelo no es incompatible con la inclusión de factores socioculturales e históricos para su análisis. Por este motivo, sería deseable que próximas investigaciones psicológicas contemplen estas perspectivas en sus indagaciones. Asimismo, en este sentido, es importante considerar que el instrumento supone un nivel de análisis descriptivo de los rasgos de la personalidad. Resulta interesante notar que, tal como se ha expuesto en este trabajo, los rasgos han sido valorados positiva o negativamente en la literatura psicológica. Próximos trabajos podrían detenerse a revisar la valoración subjetiva que los investigadores establecen respecto de qué rasgos resultan «deseables» o «indeseables» de acuerdo al contexto histórico y social de producción de conocimiento científico.

\section{Conflicto de intereses}

Los autores declaran no tener ningún conflicto de intereses.

\section{Responsabilidad ética}

Los proyectos UBACyT y PICT consignados en el trabajo son evaluados éticamente por la Universidad de Buenos Aires como requisito previo a la aprobación del subsidio. Los estudiantes fueron invitados a colaborar voluntariamente en el proyecto de investigación en el que se enmarca el presente estudio, solicitándoles su consentimiento. A lo largo de la administración de los cuestionarios, los investigadores permanecieron en presencia de los participantes. Antes de responder los cuestionarios, se les hizo constar a los participantes que los datos derivados de la investigación se utilizarían con fines exclusivamente académico-científicos bajo la Ley Nacional 25.326 de protección de los datos personales. Una vez completados los instrumentos de evaluación, estos fueron entregados a los investigadores en un sobre cerrado, con el propósito de respetar su anonimato.

\section{Contribución de autoría}

HS: concepción y diseño del estudio, interpretación de los datos y revisión final del manuscrito.

LBD: interpretación de los datos y revisión final del manuscrito.

SA: interpretación de los datos y revisión final del manuscrito. 


\section{Agradecimientos}

Este trabajo fue financiado en el marco de los proyectos de investigación UBACyT 20020170200395BA, UBACyT 20020150100232BA, PICT-2016-4147 y PICT-2014- 3432 acreditados por la Universidad de Buenos Aires (UBA) y la Agencia Nacional de Promoción Científica y Tecnológica (ANPCyT), y dirigidos por el Dr. Hugo Simkin y la Dra. Susana Azzollini. Se agradece muy especialmente al Dr. Frederick L. Oswald por brindar su autorización para la adaptación al español del Compendio Internacional de Ítems de Personalidad Abreviado Mini-IPIP.

\section{Referencias}

Allsopp, J. F., \& Feldman, M. P. (1974). Extraversion, neuroticism, psychoticism and antisocial behavior in schoolgirls. Social Behavior and Personality: An International Journal, 2(2), 184-190. doi: 10.2224/ sbp.1974.2.2.184

Aluja, A., García, Ó., \& García, L. F. (2003). Relationships among extraversion, openness to experience, and sensation seeking. Personality and Individual Differences, 35(3), 671-680. doi: 10.1016/S01918869(02)00244-1

Anderson, C., John, O. P., \& Keltner, D. (2012). The Personal Sense of Power. Journal of Personality, 80(2), 313-344. doi: 10.1111/j.1467-6494.2011.00734.x

Arthur, W., \& Graziano, W. G. (1996). The Five-Factor Model, Conscientiousness, and Driving Accident Involvement. Journal of Personality, 64(3), 593-618. doi: 10.1111/j.1467-6494.1996.tb00523.x

Ashton, M. C., \& Lee, K. (2001). A theoretical basis for the major dimensions of personality. European Journal of Personality, 15(5), 327-353. doi: 10.1002/ per.417

Ashton, M. C., Lee, K., \& Paunonen, S. V. (2002). What is the central feature of extraversion? Social attention versus reward sensitivity. Journal of Personality and Social Psychology, 83(1), 245-252. doi: 10.1037/00223514.83.1.245
Ato, M., López, J. J., \& Benavente, A. (2013). Un sistema de clasificación de los diseños de investigación en Psicología. Anales de Psicología, 29(3), 1038-1059. doi: 10.6018/analesps.29.3.178511

Baldasaro, R. E., Shanahan, M. J., \& Bauer, D. J. (2013). Psychometric Properties of the Mini-IPIP in a Large, Nationally Representative Sample of Young Adults. Journal of Personality Assessment, 95(1), 74-84. doi: 10.1080/00223891.2012.700466

Barbaranelli, C., Caprara, G. V., Vecchione, M., \& Fraley, C. R. (2007). Voters' personality traits in presidential elections. Personality and Individual Differences, 42(7), 1199-1208. doi: 10.1016/j.paid.2006.09.029

Barrera-Barrera, R., Navarro-García, A., \& Peris-Ortiz, M. (2015). El papel de la invarianza factorial en la validación del constructo calidad de servicio electrónico. Revista Europea de Dirección y Economía de la Empresa, 24(1), 13-24. doi: 10.1016/ j.redee.2014.07.001

Beauducel, A., \& Herzberg, P. Y. (2006). On the Performance of Maximum Likelihood Versus Means and Variance Adjusted Weighted Least Squares Estimation in CFA. Structural Equation Modeling: A Multidisciplinary Journal, 13(2), 186-203. doi: 10.1207/ s15328007sem1302_2

Bentler, P. M. (1990). Comparative fit indexes in structural models. Psychological Bulletin, 107(2), 238-246. doi: 10.1037/0033-2909.107.2.238

Bond, M. H. (1994). Trait Theory and Cross-Cultural Studies of Person Perception. Psychological Inquiry, 5(2), 114-117. doi: 10.1207/s15327965pli0502_2

Borders, J. (2012). Relationship Between Personality and Video Game Preferences. (Tesis de maestría). Universidad Estatal de California, EE. UU. Recuperado de http://hdl.handle.net/10211.9/1807

Bradley, B. H., Baur, J. E., Banford, C. G., \& Postlethwaite, B. E. (2013). Team Players and Collective Performance: How Agreeableness Affects Team Performance Over Time. Small Group Research, 44(6), 680-711. doi: 10.117 7/1046496413507609

Bresin, K., Hilmert, C. J., Wilkowski, B. M., \& Robinson, M. D. (2012). Response speed as an individual difference: Its role in moderating the agreeableness- 
anger relationship. Journal of Research in Personality, 46(1), 79-86. doi: 10.1016/j.jrp.2011.12.007

Breslau, N., \& Schultz, L. (2013). Neuroticism and posttraumatic stress disorder: a prospective investigation. Psychological Medicine, 43(08), 1697-1702. doi: 10.1017/S0033291712002632

Brown, T. (2015). Confirmatory factor analysis for applied research (2 ${ }^{\text {da }}$ ed.). New York: Guilford Press.

Butler, J. C. (2000). Personality and emotional correlates of right-wing authoritarianism. Social Behavior and Personality: An International Journal, 28(1), 1-14. doi: 10.2224/sbp.2000.28.1.1

Butrus, N., \& Witenberg, R. T. (2013). Some Personality Predictors of Tolerance to Human Diversity: The Roles of Openness, Agreeableness, and Empathy. Australian Psychologist, 48(4), 290-298. doi: 10.1111/j.17429544.2012.00081.x

Campo-Arias, A., \& Oviedo, H. C. (2008). Propiedades psicométricas de una escala: la consistencia interna. Revista de Salud Pública, 10(5), 831-839. Recuperado de https://www.redalyc.org/articulo.oa?id=422/ 42210515

Cao, F., Su, L., Liu, T., \& Gao, X. (2007). The relationship between impulsivity and Internet addiction in a sample of Chinese adolescents. European Psychiatry, 22(7), 466-471. doi: 10.1016/j.eurpsy.2007.05.004

Caprara, G. V., Vecchione, M., \& Schwartz, S. H. (2009). Mediational role of values in linking personality traits to political orientation. Asian Journal of Social Psychology, 12(2), 82-94. doi: 10.1111/j.1467839X.2009.01274.x

Carlo, G., Okun, M. A., Knight, G. P., \& Guzman, M. R. T. de (2005). The interplay of traits and motives on volunteering: agreeableness, extraversion and prosocial value motivation. Personality and Individual Differences, 38(6), 1293-1305. doi: 10.1016/ j.paid.2004.08.012

Carrigan, P. M. (1960). Extraversion-introversion as a dimension of personality: A reappraisal. Psychological Bulletin, 57(5), 329-360. doi: 10.1037/h0045451

Carrillo, J. M., Rojo, N., Sánchez-Bernardos, M. L., \& Avia, M. D. (2001). Openness to Experience and Depression.
European Journal of Psychological Assessment, 17(2), 130-136. doi: 10.1027//1015-5759.17.2.130

Cattell, R. B. (1950). Personality: A Systematic Theoretical and Factual Study. New York: Mc Graw Hill.

Cheung, F. M., Cheung, S. F., Leung, K., Ward, C., \& Leong, F. (2003). The English Version of the Chinese Personality Assessment Inventory. Journal of CrossCultural Psychology, 34(4), 433-452. doi: 10.1177/ 0022022103034004004

Chiorri, C., Marsh, H. W., Ubbiali, A., \& Donati, D. (2016). Testing the factor structure and measurement invariance across gender of the Big Five Inventory through exploratory structural equation modeling. Journal of Personality Assessment, 98(1), 8899. doi: 10.1080/00223891.2015.1035381

Clark, R., \& DeYoung, C. (2014). Creativity and the aspects of neuroticism. Personality and Individual Differences, 60(2014), S54. doi: 10.1016/j.paid.2013.07.224

Connelly, B. S., Ones, D. S., \& Chernyshenko, O. S. (2014). Introducing the Special Section on Openness to Experience: Review of Openness Taxonomies, Measurement, and Nomological Net. Journal of Personality Assessment, 96(1), 1-16. doi: 10.1080/ 00223891.2013.830620

Cooper, A. J., Smillie, L. D., \& Corr, P. J. (2010). A confirmatory factor analysis of the Mini-IPIP five-factor model personality scale. Personality and Individual Differences, 48(5), 688-691. doi: 10.1016/ j.paid.2010.01.004

Costa, P. T., \& McCrae, R. R. (1980a). Influence of extraversion and neuroticism on subjective well-being: Happy and unhappy people. Journal of Personality and Social Psychology, 38(4), 668-678. doi: 10.1037/ 0022-3514.38.4.668

Costa, P. T., \& McCrae, R. R. (1980b). Still stable after all these years: Personality as a key to some issues in adulthood and old age. In P. B. Baltes \& O. G. Brim (Eds.), Life span development and behavior (Vol. 3, pp. 65-102). New York: Academic Press.

Costa, P. T., \& McCrae, R. R. (1985). The NEO personality inventory manual. Odessa: Psychological Assessment Resources. 
Costa, P. T., \& McCrae, R. R. (1987). Neuroticism, Somatic Complaints, and Disease: Is the Bark Worse than the Bite? Journal of Personality, 55(2), 299-316. doi: 10.1111/j.1467-6494.1987.tb00438.x

Costa, P. T., \& McCrae, R. R. (1996). Mood and Personality in adulthood. In C. Magao \& S. H. McFadden (Eds.), Handbook of Emotion, Adult Development, and Aging (pp. 369-383). San Diego: Academic Press.

Costa, P. T., \& McCrae, R. R. (2006). Trait and factor theories. In J. C. Thomas, D. L. Segal \& M. Hersen (Eds.), Comprehensive Handbook of Personality and Psychopathology (Vol. 1, pp. 96-115). New Jersey: John Wiley \& Sons.

Costa, P. T., McCrae, R. R., \& Arenberg, D. (1980). Enduring dispositions in adult males. Journal of Personality and Social Psychology, 38(5), 793-800. doi: 10.1037/00223514.38.5.793

Costa, P. T., McCrae, R. R., \& Holland, J. L. (1984). Personality and vocational interests in an adult sample. Journal of Applied Psychology, 69(3), 390-400. doi: 10.1037/0021-9010.69.3.390

Costa, P. T., McCrae, R. R., \& Norris, A. H. (1981). Personal adjustment to aging: longitudinal prediction from neuroticism and extraversion. Journal of Gerontology, 36(1), 78-85. doi: 10.1093/geronj/36.1.78

Cullen, J. M., Wright, L. W., \& Alessandri, M. (2002). The Personality Variable Openness to Experience as It Relates to Homophobia. Journal of Homosexuality, 42(4), 119-134. doi: 10.1300/J082v42n04_08

Davidov, E., Schmidt, P., Billiet, J., \& Meuleman, B. (Eds.). (2018). Cross-Cultural Analysis: Methods and Applications ( $2^{\text {da }}$ ed.). New York: Routdledge. doi: 10.4324/9781315537078

Dermody, S. S., Wright, A. G. C., Cheong, J. W., Miller, K. G., Muldoon, M. F., Flory, J. D., ... Manuck, S. B. (2016). Personality Correlates of Midlife Cardiometabolic Risk: The Explanatory Role of HigherOrder Factors of the Five-Factor Model. Journal of Personality, 84(6), 765-776. doi: 10.1111/jopy.12216

DeYoung, C. G. (2006). Higher-order factors of the Big Five in a multi-informant sample. Journal of Personality and Social Psychology, 91(6), 1138-1151. doi: 10.1037/00223514.91.6.1138
Digman, J. M. (1990). Personality Structure: Emergence of the Five-Factor Model. Annual Review of Psychology, 41, 417-440. doi: 10.1146/annurev.ps.41.020190.002221

Digman, J. M. (1997). Higher-order factors of the Big Five. Journal of Personality and Social Psychology, 73(6), 1246-1256. doi: 10.1037/0022-3514.73.6.1246

Digman, J. M., \& Takemoto-Chock, N. K. (1981). Factors In The Natural Language Of Personality: Re-Analysis, Comparison, And Interpretation Of Six Major Studies. Multivariate Behavioral Research, 16(2), 149-170. doi: 10.1207/s15327906mbr1602_2

Dollinger, S. J. (1993). Research Note: Personality and Music Preference: Extraversion and Excitement Seeking or Openness to Experience? Psychology of Music, 21(1), 73-77. doi: 10.1177/030573569302100105

Dollinger, S. J., Leong, F. T. L., \& Ulicni, S. K. (1996). On Traits and Values: With Special Reference to Openness to Experience. Journal of Research in Personality, 30(1), 23-41. doi: 10.1006/jrpe.1996.0002

Donnellan, M. B., Oswald, F. L., Baird, B. M., \& Lucas, R. E. (2006). The mini-IPIP scales: tiny-yet-effective measures of the Big Five factors of personality. Psychological Assessment, 18(2), 192-203. doi: 10.1037/ 1040-3590.18.2.192

Duckitt, J., \& Sibley, C. G. (2010). Personality, Ideology, Prejudice, and Politics: A Dual-Process Motivational Model. Journal of Personality, 78(6), 1861-1894. doi: 10.1111/j.1467-6494.2010.00672.x

Dunn, T. J., Baguley, T., \& Brunsden, V. (2014). From alpha to omega: A practical solution to the pervasive problem of internal consistency estimation. British Journal of Psychology, 105(3), 399-412. doi: 10.1111/bjop.12046

Ekehammar, B., Akrami, N., Gylje, M., \& Zakrisson, I. (2004). What matters most to prejudice: Big Five personality, Social Dominance Orientation, or Right-Wing Authoritarianism? European Journal of Personality, 18(6), 463-482. doi: 10.1002/per.526

Eysenck, H. J. (1952). The scientific study of personality. London: Routledge \& Kegan Paul.

Eysenck, H. J., \& Eysenck, S. B. G. (1976). Psychoticism as a dimension of personality. London: Stoughton \& Hodder. 
Feist, G. J. (1998). A Meta-Analysis of Personality in Scientific and Artistic Creativity. Personality and Social Psychology Review, 2(4), 290-309. doi: 10.1207/ s15327957pspr0204_5

Feist, G. J., \& Brady, T. R. (2004). Openness to Experience, Non-Conformity, and the Preference for Abstract Art. Empirical Studies of the Arts, 22(1), 77-89. doi: 10.2190/ Y7CA-TBY6-V7LR-76GK

Fiske, D. W. (1949). Consistency of the factorial structures of personality ratings from different sources. The Journal of Abnormal and Social Psychology, 44(3), 329-344. doi: 10.1037/h0057198

Fleeson, W., \& Jayawickreme, E. (2015). Whole Trait Theory. Journal of Research in Personality, 56, 8292. doi: 10.1016/j.jp.2014.10.009

Flynn, F. J. (2005). Having an Open Mind: The Impact of Openness to Experience on Interracial Attitudes and Impression Formation. Journal of Personality and Social Psychology, 88(5), 816-826. doi: 10.1037/00223514.88.5.816

Freud, S. (1933). New introductory lectures in psychoanalysis. New York: Norton.

George, J. M., \& Zhou, J. (2001). When openness to experience and conscientiousness are related to creative behavior: an interactional approach. The Journal of Applied Psychology, 86(3), 513-524. doi: 10.1037/0021-9010.86.3.513

Goldenberg, J. L., Pyszczynski, T., McCoy, S. K., Greenberg, J., \& Solomon, S. (1999). Death, sex, love, and neuroticism: Why is sex such a problem? Journal of Personality and Social Psychology, 77(6), 11731187. doi: 10.1037/0022-3514.77.6.1173

Graziano, W. G. (1994). The development of agreeableness as a dimension of personality. In C. F. Halverson, G. A. Kohnstamm \& R. P. Martin (Eds.), The Developing Structure of Temperament and Personality From Infancy to Adulthood (pp. 339-354). Lawrence Erlbaum Associates.

Graziano, W. G., Habashi, M. M., Sheese, B. E., \& Tobin, R. M. (2007). Agreeableness, empathy, and helping: A person $\times$ situation perspective. Journal of Personality and Social Psychology, 93(4), 583-599. doi: 10.1037/ 0022-3514.93.4.583
Graziano, W. G., Jensen-Campbell, L. A., \& Hair, E. C. (1996). Perceiving interpersonal conflict and reacting to it: The case for agreeableness. Journal of Personality and Social Psychology, 70(4), 820-835. doi: 10.1037/0022-3514.70.4.820

Graziano, W. G., \& Tobin, R. M. (2009). Agreeableness. In M. R. Leary \& R. Hoyle (Eds.), Handbook of individual differences in social behavior (pp. 46-61). New York: The Guilford Press.

Green, J. R. (1994). Theatre in Ancient Greek Society. London: Routledge.

Green, S. B., \& Yang, Y. (2015). Evaluation of Dimensionality in the Assessment of Internal Consistency Reliability: Coefficient Alpha and Omega Coefficients. Educational Measurement: Issues and Practice, 34(4), 14-20. doi: 10.1111/emip.12100

Griffin, B., \& Hesketh, B. (2004). Why Openness to Experience is not a Good Predictor of Job Performance. International Journal of Selection and Assessment, 12(3), 243-251. doi: 10.1111/j.0965-075X.2004.278_1.x

Gunthert, K. C., Cohen, L. H., \& Armeli, S. (1999). The role of neuroticism in daily stress and coping. Journal of Personality and Social Psychology, 77(5), 1087-1100. doi: 10.1037/0022-3514.77.5.1087

Haas, B. W., Omura, K., Constable, R. T., \& Canli, T. (2007). Is Automatic Emotion Regulation Associated With Agreeableness?: A Perspective Using a Social Neuroscience Approach. Psychological Science, 18(2), 130-132. doi: 10.1111/j.1467-9280.2007.01861.x

Harris, J. A. (2004). Measured intelligence, achievement, openness to experience, and creativity. Personality and Individual Differences, 36(4), 913-929. doi: 10.1016/S0191-8869(03)00161-2

Heaven, P. C. L., \& Bucci, S. (2001). Right-wing authoritarianism, social dominance orientation and personality: an analysis using the IPIP measure. European Journal of Personality, 15(1), 49-56. doi: 10.1002/per.389

Heaven, P. C. L., Mulligan, K., Merrilees, R., Woods, T., \& Fairooz, Y. (2001). Neuroticism and conscientiousness as predictors of emotional, external, and restrained eating behaviors. International Journal of Eating Disorders, 30(2), 161-166. doi: 10.1002/eat.1068 
Hettema, J., Neale, M. C., Myers, J. M., Prescott, C. A., Kendler, K. S. (2006). A Population-Based Twin Study of the Relationship Between Neuroticism and Internalizing Disorders. American Journal of Psychiatry, 163(5), 857-864. doi: 10.1176/appi.ajp.163.5.857

Heuchert, J. W. P., Parker, W. D., Stumpf, H., \& Myburgh, C. P. H. (2000). The Five-Factor Model of Personality in South African College Students. American Behavioral Scientist, 44(1), 112-125. doi: 10.1177/00027640021956125

Hiel, A. van, Kossowska, M., \& Mervielde, I. (2000). The relationship between Openness to Experience and political ideology. Personality and Individual Differences, 28(4), 741-751. doi: 10.1016/S01918869(99)00135-X

Hiel, A. van, \& Mervielde, I. (2004). Openness to experience and boundaries in the mind: Relationships with cultural and economic conservative beliefs. Journal of Personality, 72(4), 659-686. doi: 10.1111/ j.0022-3506.2004.00276.x

Hills, P., \& Argyle, M. (2001a). Emotional stability as a major dimension of happiness. Personality and Individual Differences, 31(8), 1357-1364. doi: 10.1016/ S0191-8869(00)00229-4

Hills, P. R., \& Argyle, M. (2001b). Happiness, introversionextraversion and happy introverts. Personality and Individual Differences, 30(4), 595-608. doi: 10.1016/ S0191-8869(00)00058-1

Hirsh, J. B., DeYoung, C. G., Xu, X., \& Peterson, J. B. (2010). Compassionate Liberals and Polite Conservatives: Associations of Agreeableness With Political Ideology and Moral Values. Personality and Social Psychology Bulletin, 36(5), 655-664. doi: 10.1177/0146167210366854

Hodson, G., Hogg, S. M., \& MacInnis, C. C. (2009). The role of 'dark personalities' (narcissism, Machiavellianism, psychopathy), Big Five personality factors, and ideology in explaining prejudice. Journal of Research in Personality, 43(4), 686-690. doi: 10.1016/ j.jrp.2009.02.005

Holgado-Tello, F. P., Chacón-Moscoso, S., Barbero-García, I., \& Vila-Abad, E. (2009). Polychoric versus Pearson correlations in exploratory and confirmatory factor analysis of ordinal variables. Quality and Quantity, 44(1), 153-166. doi: 10.1007/s11135-008-9190-y
Hong, I., \& Rust, J. (1989). Androgyny and openness to experience in a Chinese population. Social Behavior and Personality: An International Journal, 17(2), 215218. doi: 10.2224/sbp.1989.17.2.215

Hu, L., \& Bentler, P. M. (1999). Cutoff criteria for fit indexes in covariance structure analysis: Conventional criteria versus new alternatives. Structural Equation Modeling: A Multidisciplinary Journal, 6(1), 1-55. doi: 10.1080/10705519909540118

Huang, J. L., Cropanzano, R., Li, A., Shao, P., Zhang, X., \& Li, Y. (2017). Employee Conscientiousness, Agreeableness, and Supervisor Justice Rule Compliance: A Three-Study Investigation. Journal of Applied Psychology, 102(11), 1564-1589. doi: 10.1037/ apl0000248

Jeronimus, B. F., Riese, H., Sanderman, R., \& Ormel, J. (2014). Mutual reinforcement between neuroticism and life experiences: A five-wave, 16-year study to test reciprocal causation. Journal of Personality and Social Psychology, 107(4), 751-764. doi: 10.1037/a0037009

John, O. P., Naumann, L., \& Soto, C. J. (2008). Paradigm Shift to the Integrative Big-Five Trait Taxonomy: History, Measurement, and Conceptual Issues. In O. P. John, R. W. Robins, \& L. A. Pervin (Eds.), Handbook of personality: Theory and research (pp. 114-158). New York: The Guilford Press.

John, O. P., Robins, R. W., \& Pervin, L. A. (Eds.). (2010). Handbook of Personality: Theory and Research (3 ${ }^{\text {ra }}$ ed.). New York: The Guilford Press.

John, O. P., \& Srivastava, S. (1999). The Big Five trait taxonomy: History, measurement, and theoretical perspectives. Handbook of Personality: Theory and Research, 2(510), 102-138. doi: citeulike-articleid:3488537

Jost, J. T., Nosek, B. A., \& Gosling, S. D. (2008). Ideology: Its Resurgence in Social, Personality, and Political Psychology. Perspectives on Psychological Science, 3(2), 126-136. doi: 10.1111/j.1745-6916.2008.00070.x

Judge, T. A., \& Erez, A. (2007). Interaction and intersection: The constellation of emotional stability and extraversion in predicting performance. Personnel Psychology, 60(3), 573-596. doi: 10.1111/j.17446570.2007.00084.x 
Judge, T. A., Livingston, B. A., \& Hurst, C. (2012). Do nice guys-and gals-really finish last? The joint effects of sex and agreeableness on income. Journal of Personality and Social Psychology, 102(2), 390-407. doi: 10.1037/a0026021

Judge, T. A., Vianen, A. E. M. van, \& Pater, I. E. de (2004). Emotional Stability, Core Self-Evaluations, and Job Outcomes: A Review of the Evidence and an Agenda for Future Research. Human Performance, 17(3), 325346. doi: 10.1207/s15327043hup1703_4

Jung, C. G. (1923). Psychological types: or the psychology of individuation. Oxford: Harcourt, Brace \& World.

Jung, C. G. (1939). The Integration of the Personality. Postgraduate medical journal, (Vol. 17). Oxford: Farrar \& Rinehart. doi: 10.2307/2085562

Jylhä, P., \& Isometsä, E. (2006). The relationship of neuroticism and extraversion to symptoms of anxiety and depression in the general population. Depression and Anxiety, 23(5), 281-289. doi: 10.1002/da.20167

Kaiser, R. T., \& Ozer, D. J. (1997). Emotional stability and goal-related stress. Personality and Individual Differences, 22(3), 371-379. doi: 10.1016/S01918869(96)00208-5

Kassin, S. (2003). Psychology. New York: Prentice-Hall.

Khan, A. A., Jacobson, K. C., Gardner, C. O., Prescott, C. A., \& Kendler, K. S. (2005). Personality and comorbidity of common psychiatric disorders. The British Journal of Psychiatry, 186(3), 190-196. doi: 10.1192/bjp.186.3.190

Khoo, S., \& Simms, L. J. (2018). Links between depression and openness and its facets: openness and depression. Personality and mental health, 12(3), 203215. doi: 10.1002/pmh.1417

Kline, R. B. (2005). Structural equation modeling. New York: The Guilford Press.

Kline, R. B. (2010). Principles and practice of structural equation modeling. New York: The Guilford Press.

Krueger, R. F., Caspi, A., Moffitt, T. E., Silva, P. A., \& McGee, R. (1996). Personality traits are differentially linked to mental disorders: A multitrait-multidiagnosis study of an adolescent birth cohort. Journal of Abnormal Psychology, 105(3), 299-312. doi: 10.1037/ 0021-843X.105.3.299
Laher, S. (2013). Understanding the Five-Factor Model and Five-Factor Theory through a South African cultural lens. South African Journal of Psychology, 43(2), 208221. doi: $10.1177 / 0081246313483522$

Laverdière, O., Morin, A. J. S., \& St-Hilaire, F. (2013). Factor structure and measurement invariance of a short measure of the Big Five personality traits. Personality and Individual Differences, 55(7), 739-743. doi: 10.1016/j.paid.2013.06.008

Lee, K., \& Ashton, M. C. (2012). Getting mad and getting even: Agreeableness and Honesty-Humility as predictors of revenge intentions. Personality and Individual Differences, 52(5), 596-600. doi: 10.1016/ j.paid.2011.12.004

Lee, K., Ashton, M. C., Ogunfowora, B., Bourdage, J. S., \& Shin, K. H. (2010). The personality bases of sociopolitical attitudes: The role of Honesty-Humility and Openness to Experience. Journal of Research in Personality, 44(1), 115-119. doi: 10.1016/j.jrp.2009.08.007

LePine, J. A., Colquitt, J. A., \& Erez, A. (2000). Adaptability to changing task contexts/: Effects of general cognitive ability, conscientiousness, and openness to experience. Personnel Psychology, 53(3), 563-593. doi: 10.1111/ j.1744-6570.2000.tb00214.x

Lévy, J. P. \& González, N. (2006). Modelización y causalidad. In J. P. Lévy \& J. Varela (Eds.), Modelización con estructuras de covarianzas en ciencias sociales (pp. 155-175). La Coruña, España: Netbiblo.

Lévy, J. P. \& Varela, J. (2006). Modelización con estructuras de covarianzas en ciencias sociales. La Coruña, España: Netbiblio.

Li, W., Li, X., Huang, L., Kong, X., Yang, W., Wei, D., ... Liu, J. (2015). Brain structure links trait creativity to openness to experience. Social Cognitive and Affective Neuroscience, 10(2), 191-198. doi: 10.1093/ scan/nsu041

Li, Z., Sang, Z., Wang, L., \& Shi, Z. (2012). The Mini-IPIP Scale: Psychometric Features and Relations with PTSD Symptoms of Chinese Earthquake Survivors. Psychological Reports, 111(2), 641-651. doi: 10.2466/ 16.12.15.PR0.111.5.641-651

Lucas, R. E., \& Baird, B. M. (2004). Extraversion and emotional reactivity. Journal of Personality and Social 
Psychology, 86(3), 473-485. doi: 10.1037/00223514.86.3.473

Magnus, K., Diener, E., Fujita, F., \& Pavot, W. (1993). Extraversion and neuroticism as predictors of objective life events: A longitudinal analysis. Journal of Personality and Social Psychology, 65(5), 1046-1053. doi: 10.1037/0022-3514.65.5.1046

Martínez-Molina, A., \& Arias, V. B. (2018). Balanced and positively worded personality short-forms: Mini-IPIP validity and cross-cultural invariance. PeerJ, 6, e5542. doi: 10.7717/peerj.5542

Martsh, C. T., \& Miller, W. R. (1997). Extraversion predicts heavy drinking in college students. Personality and Individual Differences, 23(1), 153-155. doi: 10.1016/ S0191-8869(97)00015-9

McAdams, D. P. (1997). A Conceptual History of Personality Psychology. In R. Hogan, J. A. Johnson, \& S. R. Briggs (Eds.), Handbook of Personality Psychology (pp. 3-39). Academic Press. doi: 10.1016/ B978-012134645-4/50002-0

McAdams, D. P., Shiner, R. L., \& Tackett, J. L. (Eds.). (2019). The Handbook of Personality Development. New York: Guilford Press.

McCrae, R. R. (1987). Creativity, divergent thinking, and openness to experience. Journal of Personality and Social Psychology, 52(6), 1258-1265. doi: 10.1037/00223514.52.6.1258

McCrae, R. R. (1993). Openness to Experience as a Basic Dimension of Personality. Imagination, Cognition and Personality, 13(1), 39-55. doi: 10.2190/H8H6-QYKRKEU8-GAQ0

McCrae, R. R. (1994). Openness to Experience: Expanding the boundaries of Factor V. European Journal of Personality, 8(4), 251-272. doi: 10.1002/per.2410080404

McCrae, R. R., \& Costa, P. T. (1996). Toward a New Generation of Personality Theories: Theoretical Contexts for the Five-Factor Model. In J. S. Wiggins (Ed.), The five-factor model of personality: Theoretical perspectives (pp. 51-87). New York: The Guilford Press.

McCrae, R. R., \& Costa, P. T. (1997). Conceptions and correlates of openness to experience. In R. Hogan, J. A. Johnson, \& S. R. Briggs (Eds.), Handbook of Personality Psychology (pp. 825-847). San Diego:
Academic Press. doi: 10.1016/B978-012134645-4/ 50032-9

McCrae, R. R., \& Costa, P. T. (2008). Empirical and theoretical status of the five-factor model of personality traits. In G. J. Boyle, G. Mathews, \& D. H. Saklofske (Eds.), The SAGE Handbook of Personality Theory and Assessment (pp. 273-294). London: Sage Publications. doi: 10.4135/9781849200462.n13

McCrae, R. R., \& Costa, P. T. (2010). The Five Factor Theory of Personality. In O. P. John, R. W. Robins, \& L. A. Pervin (Eds.), Handbook of personality: Theory and research (pp. 159-182). New York: The Guilford Press.

McCrae, R. R., \& Costa, P. T. (2012). Personality in adulthood ( $2^{\mathrm{da}}$ ed.). New York: The Guilford Press.

McCrae, R. R., \& Costa, P. T. (2013). Introduction to the empirical and theoretical status of the five-factor model of personality traits. In G. J. Boyle, G. Mathews, \& D. H. Saklofske (Eds.), Personality disorders and the fivefactor model of personality, (3ra ed., pp. 15-27). Washington: American Psychological Association. doi: 10.1037/13939-002

McCrae, R. R., \& John, O. P. (1992). An introduction to the five-factor model and its applications. Journal of Personality, 60(2), 175-215. doi: 10.1111/j.14676494.1992.tb00970.x

McCrae, R. R., \& Sutin, A. R. (2009). Openness to Experience. In M. R. Leary \& R. H. Hoyle (Eds.), Handbook of individual differences in social behavior (pp. 257-273). New York: The Guilford Press.

McCrae, R. R., \& Sutin, A. R. (2018). A Five-Factor Theory Perspective on Causal Analysis. European Journal of Personality, 32(3), 151-166. doi: 10.1002/per.2134

McCrae, R. R., Yamagata, S., Jang, K. L., Riemann, R., Ando, J., Ono, Y., ... Spinath, F. M. (2008). Substance and artifact in the higher-order factors of the Big Five. Journal of Personality and Social Psychology, 95(2), 442-455. doi: 10.1037/a0013545

Meier, B. P., \& Robinson, M. D. (2004). Does Quick to Blame Mean Quick to Anger? The Role of Agreeableness in Dissociating Blame and Anger. Personality and Social Psychology Bulletin, 30(7), 856-867. doi: 10.1177/0146167204264764 
Meredith, W. (1993). Measurement invariance, factor analysis and factorial invariance. Psychometrika, 58(4), 525-543. doi: 10.1007/BF02294825

Miller, J. D., Lynam, D., Zimmerman, R. S., Logan, T. K., Leukefeld, C., \& Clayton, R. (2004). The utility of the Five Factor Model in understanding risky sexual behavior. Personality and Individual Differences, 36(7), 1611-1626. doi: 10.1016/j.paid.2003.06.009

Montero, I., \& León, O. G. (2007). A guide for naming research studies in Psychology. International Journal of Clinical and Health Psychology, 7(3), 847-862. Recuperado de https://www.redalyc.org/articulo.oa ?id=337/33770318

Muñiz, J., Elosua, P., \& Hambleton, R. K. (2013). Directrices para la traducción y adaptación de los tests: segunda edición. Psicothema, 25(2), 151-157. doi: 10.7334/ psicothema2013.24

Muñiz, J., \& Hambleton, R. K. (2000). Adaptación de los test de unas culturas a otras. Metodología de las Ciencias del Comportamiento, 2(2), 129-149.

Norman, W. T. (1963). Toward an adequate taxonomy of personality attributes: replicated factors structure in peer nomination personality ratings. Journal of Abnormal and Social Psychology, 66(6), 574-583. doi: 10.1037/h0040291

Nowakowska, C., Strong, C. M., Santosa, C. M., Wang, P. W., \& Ketter, T. A. (2005). Temperamental commonalities and differences in euthymic mood disorder patients, creative controls, and healthy controls. Journal of Affective Disorders, 85(1-2), 207215. doi: 10.1016/j.jad.2003.11.012

Oliveira, J. P. (2017). Psychometric Properties of the Portuguese Version of the Mini-IPIP five-Factor Model Personality Scale. Current Psychology, 38, 432-439. doi: 10.1007/s12144-017-9625-5

Ong, E. Y. L., Ang, R. P., Ho, J. C. M., Lim, J. C. Y., Goh, D. H., Lee, C. S., \& Chua, A. Y. K. (2011). Narcissism, extraversion and adolescents' self-presentation on Facebook. Personality and Individual Differences, 50(2), 180-185. doi: 10.1016/j.paid.2010.09.022

Ormel, J., Jeronimus, B. F., Kotov, R., Riese, H., Bos, E. H., Hankin, B., ... Oldehinkel, A. J. (2013). Neuroticism and common mental disorders: Meaning and utility of a complex relationship. Clinical Psychology Review, 33(5), 686-697. doi: 10.1016/j.cpr.2013.04.003

Ormel, J., Rosmalen, J., \& Farmer, A. (2004). Neuroticism: a non-informative marker of vulnerability to psychopathology. Social Psychiatry and Psychiatric Epidemiology, 39(11), 906-912. doi: 10.1007/s00127-0040873-y

Packer, D. J. (2010). The interactive influence of conscientiousness and openness to experience on dissent. Social Influence, 5(3), 202-219. doi: 10.1080/ 15534511003676441

Paulus, D. J., Vanwoerden, S., Norton, P. J., \& Sharp, C. (2016). From neuroticism to anxiety: Examining unique contributions of three transdiagnostic vulnerability factors. Personality and Individual Differences, 94, 3843. doi: 10.1016/j.paid.2016.01.012

Pérez-García, A. M., \& Bermúdez-Moreno, J. (2012). Introducción al Estudio de la Personalidad: Unidades de Análisis. In J. Bermúdez-Moreno, A. M. PérezGarcía, A. Ruiz-Caballero, P. Sanjuán-Suárez, \& B. Rueda-Laffond (Eds.), Psicología de la Personalidad (pp. 25-68). Madrid: Universidad Nacional de la Educaciòn a Distancia.

Perkins, A. M., Arnone, D., Smallwood, J., \& Mobbs, D. (2015). Thinking too much: self-generated thought as the engine of neuroticism. Trends in Cognitive Sciences, 19(9), 492-498. doi: 10.1016/j.tics.2015.07.003

Perry, R., \& Sibley, C. G. (2013). Seize and freeze: Openness to Experience shapes judgments of societal threat. Journal of Research in Personality, 47(6), 677-686. doi: 10.1016/j.jrp.2013.06.006

Piedmont, R. L. (1999). Does Spirituality Represent the Sixth Factor of Personality? Spiritual Transcendence and the Five-Factor Model. Journal of Personality, 67(6), 985-1013. doi: 10.1111/1467-6494.00080

Piedmont, R. L., \& Chae, J.-H. (1997). Cross-Cultural Generalizability of the Five-Factor Model of Personality: Development and Validation of the NEO PI-R for Koreans. Journal of Cross-Cultural Psychology, 28(2), 131-155. doi: 10.1177/ 0022022197282001

Piedmont, R. L., Sherman, M. F., \& Sherman, N. C. (2012). Maladaptively high and low openness: the case for 
experiential permeability. Journal of Personality, 80(6), 1641-1668. doi: 10.1111/ j.1467-6494.2012.00777.x

Prilleltensky, I. (2003). Poverty and Power. In S. C. Carr \& T. Sloan (Eds.), International and cultural psychology series. Poverty and Psychology: From global perspective to local practice (pp. 19-44). New York: Kluwer Academic Publishers. doi: 10.1007/978-1-46150029-2_2

Rammstedt, B. (2007). Who worries and who is happy? Explaining individual differences in worries and satisfaction by personality. Personality and Individual Differences, 43(6), 1626-1634. doi: 10.1016/ j.paid.2007.04.031

Roberts, B. W., Jackson, J. J., Fayard, J. V., Edmonds, G., \& Meints, J. (2009). Conscientiousness. In M. R. Leary $\&$ R. Hoyle (Eds.), Handbook of individual differences in social behavior (pp. 257-273). New York: The Guilford Press.

Rothmann, S., \& Coetzer, E. P. (2003). The big five personality dimensions and job performance. $S A$ Journal of Industrial Psychology, 29(1), 68-74. doi: 10.4102/sajip.v29i1.88

Roy, A. Jong, J, de, Linnoila, M. (1989). Extraversion in Pathological Gamblers. Archives of General Psychiatry, 46(8), 679. doi: 10.1001/archpsyc.1989.01810080009001

Rusting, C. L., \& Larsen, R. J. (1997). Extraversion, neuroticism, and susceptibility to positive and negative affect: A test of two theoretical models. Personality and Individual Differences, 22(5), 607-612. doi: 10.1016/S0191-8869(96)00246-2

Samuel, D. B., \& Gore, W. L. (2012). Maladaptive Variants of Conscientiousness and Agreeableness. Journal of Personality, 80(6), 1669-1696. doi: 10.1111/j.14676494.2012.00770.x

Samuel, D. B., South, S. C., \& Griffin, S. A. (2015). Factorial invariance of the five-factor model rating form across gender. Assessment, 22(1), 65-75. doi: 10.1177/ 1073191114536772

Schermelleh-Engel, K., Moosbrugger, H., \& Müller, H. (2003). Evaluating the Fit of Structural Equation Models: Tests of Significance and Descriptive Goodness-of-Fit Measures. Methods of Psychological Research Online, 8(8), 23-74.
Schumacker, R. E., \& Lomax, R. G. (2004). A beginner's guide to estructural equation modeling. New Jersey: Lawrence Erlbaum Associates.

Sibley, C. G., \& Duckitt, J. (2009). Big-five personality, social worldviews, and ideological attitudes: further tests of a dual process cognitive-motivational model. The Journal of Social Psychology, 149(5), 545-561. doi: 10.1080/00224540903232308

Silvia, P. J., Nusbaum, E. C., Berg, C., Martin, C., \& O’Connor, A. (2009). Openness to experience, plasticity, and creativity: Exploring lower-order, high-order, and interactive effects. Journal of Research in Personality, 43(6), 1087-1090. doi: 10.1016/j.jp.2009.04.015

Silvia, P. J., \& Sanders, C. E. (2010). Why are smart people curious? Fluid intelligence, openness to experience, and interest. Learning and Individual Differences, 20(3), 242-245. doi: 10.1016/j.lindif.2010.01.006

Simkin, H. (2017). Adaptación y validación al español de la Escala de Evaluación de Espiritualidad y Sentimientos Religiosos (ASPIRES): la trascendencia espiritual en el modelo de los cinco factores. Universitas Psychologica, 16(2), 1-12. doi: 10.11144/Javeriana.upsy16-2.aeee

Simkin, H., \& Pérez-Marín, M. (2018). Personalidad y autoestima: un análisis sobre el importante papel de sus relaciones. Terapia Psicologica, 36(1), 15-22. doi: 10.4067/s0718-48082017000300015

Sloan, T. (1997). Theories of Personality: Ideology and Beyond. In D. Fox \& I. Prilleltensky (Eds.), Critical Psychology. An Introduction (pp. 87-103). London: Sage Publications.

Sloan, T. (2009). Theories of Personality. In Dennis Fox, I. Prilleltensky \& S. Austin (Eds.), Critical Psychology: An Introduction (2da ed., pp. 57-75). Los Angeles: Sage Publications.

Smith, K. A., Barstead, M. G., \& Rubin, K. H. (2017). Neuroticism and Conscientiousness as Moderators of the Relation Between Social Withdrawal and Internalizing Problems in Adolescence. Journal of Youth and Adolescence, 46(4), 772-786. doi: 10.1007/ s10964-016-0594-z

Srivastava, S., \& Ketter, T. A. (2010). The Link Between Bipolar Disorders and Creativity: Evidence from Personality and Temperament Studies. Current 
Psychiatry Reports, 12(6), 522-530. doi: 10.1007/s11920010-0159-x

Steiner, M., Allemand, M., \& McCullough, M. E. (2012). Do agreeableness and neuroticism explain age differences in the tendency to forgive others? Personality \& Social Psychology Bulletin, 38(4), 441453. doi: $10.1177 / 0146167211427923$

Steptoe, A., Easterlin, E., \& Kirschbaum, C. (2017). Conscientiousness, hair cortisol concentration, and health behaviour in older men and women. Psychoneuroendocrinology, 86, 122-127. doi: 10.1016/ j.psyneuen.2017.09.016

Strelan, P. (2007). Who forgives others, themselves, and situations? The roles of narcissism, guilt, self-esteem, and agreeableness. Personality and Individual Differences, 42(2), 259-269. doi: 10.1016/ j.paid.2006.06.017

Suls, J., Martin, R., \& David, J. P. (1998). PersonEnvironment Fit and its Limits: Agreeableness, Neuroticism, and Emotional Reactivity to Interpersonal Conflict. Personality and Social Psychology Bulletin, 24(1), 88-98. doi: 10.1177/0146167298241007

Sutin, A. R., Terracciano, A., Ferrucci, L., \& Costa, P. T. (2010). Teeth grinding: Is Emotional Stability related to bruxism? Journal of Research in Personality, 44(3), 402-405. doi: 10.1016/j.jp.2010.03.006

Trull, T. J., \& Durrett, C. A. (2005). Categorical and dimensional models of personality disorder. Annual Review of Clinical Psychology, 1, 355-380. doi: 10.1146/annurev.clinpsy.1.102803.144009

Tupes, E. C., \& Christal, R. E. (1961). Recurrent Personality Factors Based on Trait Ratings. Journal of Personality. Journal of Personality, 60(2), 225-251. doi: 10.1111/ j.1467-6494.1992.tb00973.x

Uziel, L. (2006). The extraverted and the neurotic glasses are of different colors. Personality and Individual Differences, 41(4), 745-754. doi: 10.1016/ j.paid.2006.03.011

Venn, C. (1984). The subject of psychology. In J. Enriques, W. Hollway, C. Urwin, C. Venn, \& W. Valerie (Eds.), Changing the Subject: Psychology, Social Regulation, and Subjectivity (pp. 119-152). London: Methuen.
Walker, D. F., \& Gorsuch, R. L. (2002). Forgiveness within the Big Five personality model. Personality and Individual Differences, 32(7), 1127-1137. doi: 10.1016/ S0191-8869(00)00185-9

Watson, D., \& Clark, L. A. (1992). On traits and temperament: general and specific factors of emotional experience and their relation to the five-factor model. Journal of Personality, 60(2), 441-476. doi: 10.1111/ j.1467-6494.1992.tb00980.x

Watson, D., \& Clark, L. A. (1997). Extraversion and Its Positive Emotional Core. In R. Hogan, J. A. Johnson, \& S. R. Briggs (Eds.), Handbook of Personality Psychology (pp. 767-793). Academic Press. doi: 10.1016/ B978-012134645-4/50030-5

Whiteside, S. P., \& Lynam, D. R. (2001). The Five Factor Model and impulsivity: using a structural model of personality to understand impulsivity. Personality and Individual Differences, 30(4), 669-689. doi: 10.1016/ S0191-8869(00)00064-7

Widiger, T. A. (2009). Neuroticism. In M. R. Leary \& R. Hoyle (Eds.), Handbook of individual differences in social behavior (pp. 129-146). New York: The Guilford Press.

Wiles, D. (2007). Mask and Performance in Greek Tragedy: From Ancient Festival to Modern Experimentation. Cambridge: Cambridge University Press.

Williams, D. G. (1992). Dispositional optimism, neuroticism, and extraversion. Personality and Individual Differences, 13(4), 475-477. doi: 10.1016/01918869(92)90076-2

Wilt, J., \& Revelle, W. (2009). Extraversion. In M. R. Leary \& R. Hoyle (Eds.), Handbook of individual differences in social behavior (pp. 257-273). New York: The Guilford Press.

Witt, L. A., Burke, L. A., Barrick, M. R., \& Mount, M. K. (2002). The interactive effects of conscientiousness and agreeableness on job performance. Journal of Applied Psychology, 87(1), 164-169. doi: 10.1037/00219010.87.1.164

Wolfestein, M., \& Trull, T. J. (1997). Depression and Openness to Experience. Journal of Personality Assessment, 69(3), 614-632. doi: 10.1207/s1532775 2jpa6903_14 
Woo, S. E., Chernyshenko, O. S., Longley, A., Zhang, Z.X., Chiu, C.-Y., \& Stark, S. E. (2014). Openness to Experience: its lower level structure, measurement, and cross-cultural equivalence. Journal of Personality Assessment, 96(1), 29-45. doi: 10.1080/00223891.2 013.806328

Yoon, K. L., Maltby, J., \& Joormann, J. (2013). A pathway from neuroticism to depression: examining the role of emotion regulation. Anxiety, Stress \& Coping, 26(5), 558-572. doi: 10.1080/10615806.2012.734810

Zoeterman, S. E., \& Wright, A. J. (2014). The Role of Openness to Experience and Sexual Identity Formation in LGB Individuals: Implications for Mental Health. Journal of Homosexuality, 61(2), 334-353. doi: 10.1080/ 00918369.2013.839919

\section{Hugo Simkin}

Facultad de Ciencias Sociales, Universidad de Buenos Aires, Argentina.

Consejo Nacional de Investigaciones Científicas y Técnicas, Argentina.

Doctor en psicología y Catedrático de su universidad. Su trabajo se centra en la relación entre la personalidad, la espiritualidad y la salud mental.

ORCID: http://orcid.org/0000-0001-7162-146X

Autor corresponsal: hugosimkin@sociales.uba.ar

Lucrecia Borchardt Duter

Facultad de Ciencias Sociales, Universidad de Buenos Aires, Argentina.

Becaria estímulo UBACyT de su universidad. Su trabajo se centra en el vínculo entre el apoyo social y la autoestima en el marco del modelo y la teoría de los cinco factores de la personalidad.

ORCID: ttp://orcid.org/0000-0003-3971-007X

borchardt@sociales.uba.ar

Susana Azzollini

Consejo Nacional de Investigaciones Científicas y Técnicas, Argentina.

Instituto de Investigaciones, Facultad de Psicología, Universidad de Buenos Aires, Argentina.

Doctora en psicología y Catedrática de su universidad. Su trabajo se centra en la relación entre la personalidad y la centralidad de los eventos traumáticos en situaciones de catástrofe.

ORCID: http://orcid.org/0000-0002-3192-5087

susanaazzollini@psi.uba.ar 


\section{Anexo}

Ítems del Inventario Mini-IPIP adaptados al contexto argentino

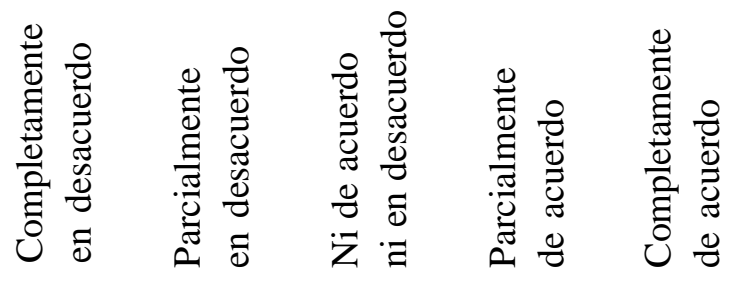

1. Soy el alma de las fiestas

2. Empatizo con lo que sienten los demás

3. Hago mis tareas de inmediato

4. Tengo cambios frecuentes en mi estado de ánimo

5. Tengo una gran imaginación

6. No suelo hablar mucho*

7. No me interesan mucho los problemas de los demás* cosas en su lugar ${ }^{*}$

9. Suelo estar relajado/a la mayor parte del tiempo*

10. No me interesan las ideas abstractas*

11. Hablo con muchas personas diferentes en las fiestas

12. Puedo sentir las emociones que sienten los demás

13. Me gusta el orden

14. Me enojo fácilmente

15. Tengo dificultad para entender ideas abstractas*

16. No me gusta llamar la atención*

17. No me interesan realmente los demás*

18. Soy algo desordenado/a*

19. Raras veces me siento triste*

20. No tengo una buena imaginación*
8. Usualmente me olvido de volver a guardar las

1

2

3

3

4

5

1

1

1

1

1

1

3

4

\section{5}

3

4

5

3

4

5

3

4

5

3

4

5

45

$\begin{array}{lllll}1 & 2 & 3 & 4 & 5 \\ 1 & 2 & 3 & 4 & 5 \\ 1 & 2 & 3 & 4 & 5 \\ 1 & 2 & 3 & 4 & 5 \\ 1 & 2 & 3 & 4 & 5 \\ 1 & 2 & 3 & 4 & 5 \\ 1 & 2 & 3 & 4 & 5 \\ 1 & 2 & 3 & 4 & 5 \\ 1 & 2 & 3 & 4 & 5 \\ 1 & 2 & 3 & 4 & 5 \\ 1 & 2 & 3 & 4 & 5 \\ 1 & 2 & 3 & 4 & 5 \\ 1 & 2 & 3 & 4 & 5\end{array}$

* Ítems invertidos 